\title{
Development of a scoring scale for predicting the risk of postoperative complications after spinal tuberculosis debridement: a retrospective cohort study of 233 patients
}

\author{
Guanyin Jiang, Yunsheng Ou, Yong Zhu, Wei Luo, Xing Du, Wei Zhang, Wanyuan Qin \\ Department of Orthopedics, The First Affiliated Hospital of Chongqing Medical University, Chongqing, China \\ Contributions: (I) Conception and design: G Jiang, Y Ou; (II) Provision of study materials and patients: X Du; (III) Collection and assembly of data: \\ Y Zhu, W Luo; (IV) Data analysis and interpretation: G Jiang; (V) Administrative support: Y Ou; (VI) Manuscript writing: All authors; (VII) Final \\ approval of manuscript: All authors. \\ Correspondence to: Yunsheng Ou. Department of Orthopedics, The First Affiliated Hospital of Chongqing Medical University, Chongqing 400016, \\ China. Email: ouyunsheng2001@163.com.
}

Background: This investigation established a scoring scale for predicting the incidence of postoperative complications in patients after spinal tuberculosis debridement.

Methods: A total of 232 spinal tuberculosis patients who underwent debridement surgery between January 2012 to May 2020 were included in this retrospective study. The study cohort was divided into 2 groups according to the presence or absence of postoperative complications. The complications were defined as abnormal or impaired body function caused by surgical factors (such as nerve injury and internal fixation looseness) or other factors (such as chemotherapy and bed stay). Clinical characteristics include age, body mass index (BMI), diabetes mellitus, pulmonary tuberculosis, smoking history, preoperative serum albumin, preoperative C-reactive protein (CRP), Cobb angle correction, surgical approach, operation time, etc. operative blood loss was recorded and analyzed. The clinical characteristics of spinal tuberculosis patients who suffered postoperative complications were evaluated, and a scoring scale was established using logistic regression analysis. The performance of this scoring scale was prospectively validated.

Results: Out of 232 patients, a total of 188 (81.03\%) suffered postoperative complications after surgery. Multivariate binary logistic regression analysis showed that diabetes mellitus [adjusted odds ratio (OR) $=1.110, \mathrm{P}=0.046$ ], pulmonary tuberculosis (adjusted $\mathrm{OR}=1.181, \mathrm{P}=0.002$ ), low preoperative serum albumin (adjusted $\mathrm{OR}=0.789, \mathrm{P}=0.005$ ), anterior surgical approach (adjusted $\mathrm{OR}=5.934, \mathrm{P}=0.035$ ), and long operation time (adjusted $\mathrm{OR}=1.019, \mathrm{P}<0.01$ ) were independent risk factors of postoperative complications after spinal tuberculosis debridement surgery. The above independent risk factors were assigned to establish a scoring scale for predicting postoperative complications, and receiver operating characteristic (ROC) analysis showed that the optimal cut-off value for the scoring scale was 4 points. The sensitivity and specificity of the scoring scale were $60.8 \%$ and $81.8 \%$, respectively, based on the validation set.

Conclusions: Using the scoring scale, spinal tuberculosis patients with a score between 4 to 9 would be considered at high risk of postoperative complications, while patients with a score of 0 to 3 would likely be at low risk of developing postoperative complications.

Keywords: Spinal tuberculosis (STB); postoperative complications; risk factors; scoring scale

Submitted Apr 09, 2021. Accepted for publication Jul 23, 2021.

doi: $10.21037 / \mathrm{apm}-21-851$

View this article at: https://dx.doi.org/10.21037/apm-21-851 


\section{Introduction}

Spinal tuberculosis (STB) is common extrapulmonary tuberculosis, accounting for about $50 \%$ of osteoarticular tuberculosis (1). At present, anti-tuberculosis (TB) drug therapy combined with surgical treatment is considered the gold standard for STB treatment (2). The debridement of lesions is a key step in STB surgery as it enhances the control of tuberculosis changes, improves the efficacy of anti-TB drugs, promotes bone graft fusion, and reduces the risk of recurrence of STB $(3,4)$.

However, debridement is an iatrogenic trauma for patients. In addition, most STB patients have comorbidities such as diabetes, anemia, and hypoalbuminemia, and thus, they have a significantly higher risk of postoperative complications than other patients with degenerative diseases (5-7). Therefore, minimizing complications after debridement is a key focus in STB surgery. Recently, research on postoperative complications of STB has been confined to specific complications such as nerve injury, postoperative intestinal obstruction, and others. However, these studies did not explore the risk factors for other complications and therefore have little significance for the prediction and risk assessment of postoperative complications in spinal tuberculosis patients. Several studies have determined that preoperative albumin values, preoperative comorbidities, and operation time are risk factors of postoperative complications in STB (7-9). These latter studies only examined the role of a single factor in predicting postoperative complications, and thus, an accurate assessment of complication risk cannot be conducted when patients have only some of these risk factors. In addition, to date, there is a paucity of large sample studies reporting the incidence of complications after STB (7-12).

This study retrospectively examined the case data of STB patients who underwent debridement surgery in our hospital to identify the risk factors of postoperative complications. The relative contribution of various risk factors was assessed using quantitative methods to establish a predictive scoring scale of postoperative complications. This scale was established to predict the risk of postoperative complications in STB patients.

We present the following article in accordance with the TRIPOD reporting checklist (available at https://dx.doi. org/10.21037/apm-21-851).

\section{Methods}

This study was conducted in accordance with the Declaration of Helsinki (as revised in 2013) and was approved by the Institutional Ethics Board of The First Affiliated Hospital of Chongqing Medical University (No. ChiCTR1800019109). All participants provided written informed consent to participate in this study.

\section{Patient selection}

A total of 232 patients with STB who underwent lesion debridement in our hospital from January 2012 to May 2020 were retrospectively included in this study.

\section{Inclusion criteria}

Patients were selected if they met the following inclusion criteria: (I) medical records were complete, including general information, preoperative laboratory examination, imaging results [including magnetic resonance imaging (MRI) and computed tomography (CT)], and clinical data on postoperative complications; (II) patients who underwent surgical treatment; and (III) lesion tissues were extracted during the surgery, and postoperative pathological diagnosis was confirmed as STB.

\section{Exclusion criteria}

Patients were excluded if they presented with the following: (I) suspected STB not confirmed by pathological examination; (II) preliminary and pathological diagnosis of diseases other than STB; or (III) a previous history of STB.

\section{Measures and outcomes}

\section{Measures}

Based on previous studies and our experience, the following possible predictors for the occurrence of postoperative complications in STB patients were assessed: patient's general conditions, laboratory examination indexes, and imaging examination indexes. Measures of general patient conditions included age, gender, height, body weight, body mass index (BMI), comorbidities, history of drinking, history of smoking, surgical approach, operation time, operative blood loss, course of disease, and postoperative hospital stay. Laboratory examination indexes included preoperative hemoglobin, preoperative serum albumin, preoperative lymphocytes, preoperative erythrocyte sedimentation rate (ESR), and preoperative C-reactive protein (CRP). Imaging examination indexes included Cobb angle correction and the number of fixation segments. Postoperative complications included operation-related complications (such as low 
serum albumin, anemia, high fever, cerebrospinal fluid leakage, delirium, delayed wound healing or infection, rupture of iliac vein, and internal fixation instability), antiTB chemotherapy-related complications (such as abnormal liver function, abnormal kidney function, and limb nerve symptoms), stay-in-bed-related complications (such as gastrointestinal symptoms, electrolyte disorders, thrombus, and urinary tract infections), and others complications (such as restricted respiratory function, respiratory failure, and pleural effusion).

\section{Development of the scoring scale}

All included patients were divided into two groups according to the presence or absence of postoperative complications (POCs). Patients experiencing POCs were assigned the "with POC group", and those without POCs were assigned into the "without POC group".

Univariate analyses were conducted on the general conditions, laboratory examination indexes, and imaging examination indexes of patients in the two groups to identify possible predictors of POCs. Multivariate logistic regression analyses were subsequently performed to identify the predictors POCs in STB patients. These indicators were then used to develop the scoring scale.

Statistically significant continuous variables (preoperative serum albumin and operation time) were converted to dichotomous variables using receiver operating characteristic (ROC) curves analysis. The weighted score of each item was determined based on the relative size of the $\mathrm{P}$-value according to the method reported by Kharbanda et al. and Zhou et al. $(13,14)$.

The appropriate cut-off points for the scoring scale were determined using ROC curves corresponding to the curve nearest the ROC graph's upper left corner.

\section{Follow up: validation of the scoring scale}

From June 2020 to March 2021, a total of 62 STB patients were enrolled to validate the accuracy of the scoring scale. The inclusion and exclusion criteria for the validation set were identical to the criteria of the derivation set.

Clinicians followed up with all patients through phone communications and outpatient consultations. No clinical data were lost from any of the included patients.

\section{Statistical analysis}

The ROC curves analysis determined both the threshold values for continuous variables and the appropriate cutoff points for the scoring scale. The prevalence of the included clinical characteristics was evaluated by calculating the sensitivity and specificity for each factor. The clinical characteristics were also subjected to univariate logistic regression analysis, and the significant factors were evaluated by multivariate logistic regression analysis. The items of the scoring system were determined by multivariate logistic regression, and the weighted score of each item was based on the relative size of the $\mathrm{P}$ value. $\mathrm{P}<0.05$ was considered statistically significant. The SPSS version 23.0 software was used for statistical analyses.

\section{Results}

\section{Patient population}

Among the 232 patients enrolled in the study, 188 patients presented with postoperative complications, including 102 males and 86 females. A total of 44 patients, including 27 males and 17 females, did not have any postoperative complications (Table 1). The various complications experienced are shown in Table 2. The mean ages of patients with and without postoperative complications were $48.75 \pm 16.32$ and $41.57 \pm 14.83$ years, respectively (Table 1 ).

\section{Results of univariate and multivariate logistic regression analysis}

Univariate logistic regression analysis found that age, BMI, diabetes mellitus, pulmonary tuberculosis, smoking history, preoperative serum albumin, preoperative CRP, Cobb angle correction, anterior surgical approach, operation time, and perioperative blood loss were all risk factors for postoperative complications (Table 3). Multivariate logistic regression analysis on the above significant risk factors revealed that diabetes mellitus, pulmonary tuberculosis, preoperative serum albumin, anterior surgical approach, and operation time were independent risk factors for the incidence of postoperative complications (Table 4). ROC curves showed that the diagnostic threshold of preoperative serum albumin was $40 \mathrm{~g} / \mathrm{L}$ (sensitivity: 0.841 ; specificity: 0.559 ) and the threshold of operation time was 181 minutes (sensitivity: 0.702, specificity: 0.659) (Figure 1).

\section{Development of the scoring scale}

The five clinical characteristics identified using the 
Table 1 Comparison of perioperative characteristics between patients with and without postoperative complications

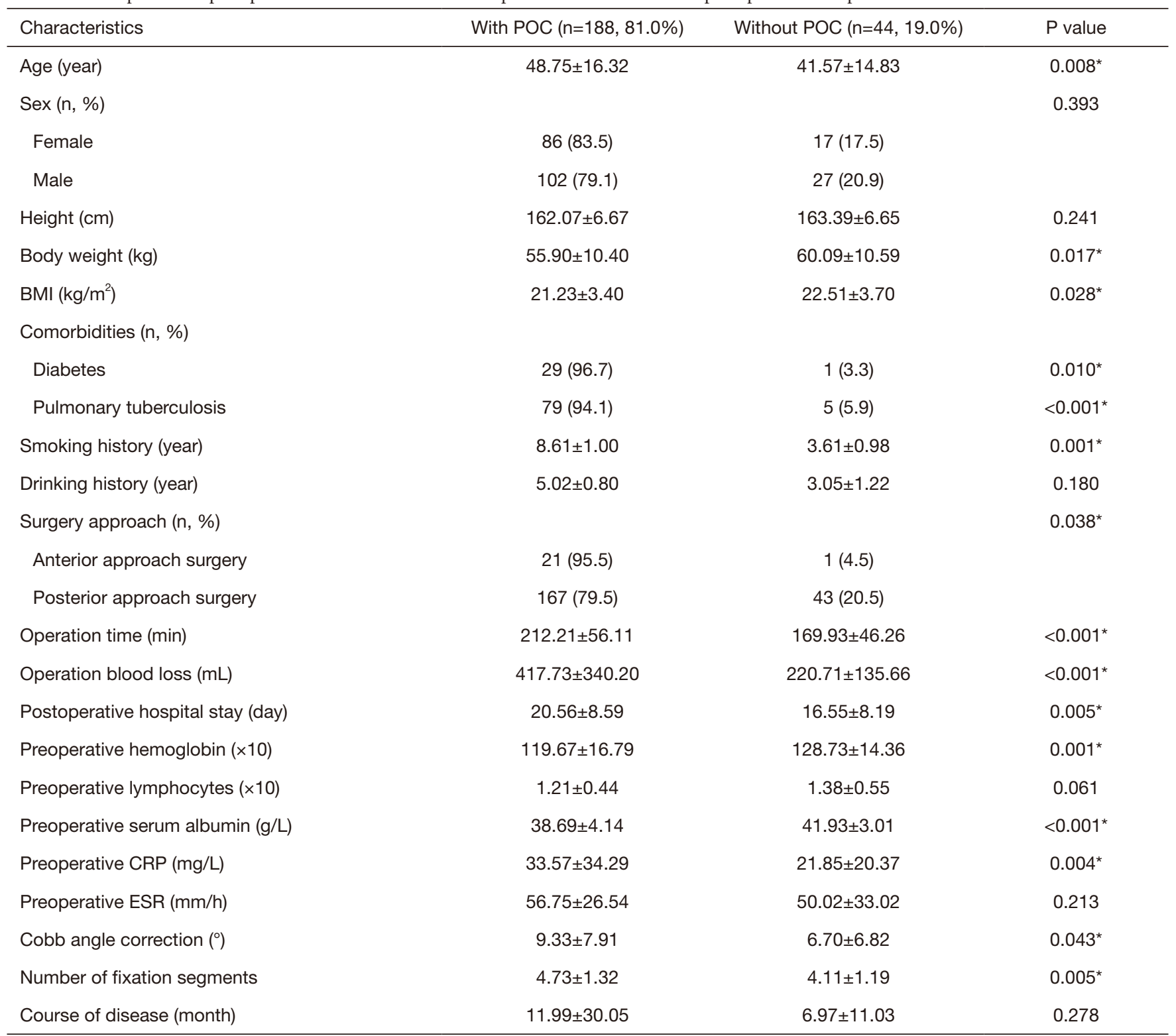

*, P<0.05. POC, postoperative complication; BMI, body mass index; CRP, C-reactive protein; ESR, erythrocyte sedimentation rate.

multivariate analysis above were used to develop the scoring scale. According to the $\mathrm{P}$ value, diabetes mellitus $(\mathrm{P}=0.046)$ and anterior surgical approach $(\mathrm{P}=0.035)$ were assigned 1 point; pulmonary tuberculosis $(\mathrm{P}=0.002)$ and preoperative serum albumin $(\mathrm{P}=0.005)$ were assigned 2 points; and operation time $(\mathrm{P}<0.001)$ was assigned 3 points (Table 5$)$. The ROC curve showed that the diagnostic threshold score of the scoring scale was 4 points (sensitivity: 0.654, specificity: 0.864) (Figure 2).

\section{Validation of the scoring scale}

The scoring scale was applied to 62 patient cases in the validation set. A comparison of the results of the score scale using the derivation set and the validation set is shown in Table 5. Based on the threshold value of 4 points, the sensitivity and specificity of the score for predicting STB postoperative complication were $65.4 \%$ and $86.4 \%$ in the derivation set, respectively, and $60.8 \%$ and $81.8 \%$ in the 
Table 2 Details of 188 patients with different postoperative complications

\begin{tabular}{lc}
\hline Complications & Number \\
\hline Total & 188 \\
Low serum albumin & 99 \\
Anemia & 72 \\
High fever & 28 \\
Gastrointestinal symptoms & 25 \\
Cerebrospinal fluid leakage & 9 \\
Electrolyte disorders & 10 \\
Abnormal liver function & 18 \\
Abnormal kidney function & 4 \\
Delirium & 2 \\
Limb nerve symptoms & 14 \\
Drug side effect & 8 \\
Thrombus & 3 \\
Urinary tract infection & 1 \\
Wound delayed healing or infection & 1 \\
Restricted respiratory function & 1 \\
Pleural effusion & 1 \\
Rupture of iliac vein & 1 \\
Internal fixation instability & 1 \\
Respiratory failure & 1 \\
\hline & \\
\hline
\end{tabular}

validation set, respectively (Table 6).

\section{Risk prediction of the scoring scale}

Based on the existing medical records of the 232 cases, the incidence of postoperative complications was obtained for each score in the score table (Table 7). A corresponding nomogram was created to facilitate the risk assessment of the scoring scale (Figure 3).

\section{Discussion}

In the present study, logistic regression analysis revealed that diabetes mellitus, pulmonary tuberculosis, low preoperative serum albumin values, anterior surgical approach, and long operation time were independent risk factors for postoperative complications in STB patients.

\section{Clinical findings: comorbidities}

Diabetes mellitus was identified as an independent risk factor for postoperative complications in STB, and STB patients complicated with diabetes were more likely to have postoperative complications. Diabetes has been associated with an increased risk of postoperative infections, postoperative blood transfusions, higher hospitalization costs, and longer hospital stays for patients who undergo spinal surgery (15), which was consistent with our findings. Sharma et al. found that patients with diabetes who underwent spinal surgery have significantly higher rates of postoperative complications and longer hospital stays (16). Previous studies on diabetes in surgical patients mainly focused on the specific clinical outcomes or complications; for example, satisfactory preoperative blood glucose management can reduce the incision infection rate and reduce the morbidity and mortality of patients in intensive care (17). In our study, blood glucose levels were maintained at $6-10 \mathrm{mmol} / \mathrm{L}$ by normative preoperative insulin regulation in all diabetic patients. Despite this, diabetes mellitus was still an independent risk factor for overall postoperative complications, suggesting that good preoperative blood glucose control in STB patients with diabetes can only reduce the occurrence of some specific complications, with little effect on the overall incidence of postoperative complications.

Pulmonary tuberculosis was also identified as an independent risk factor for postoperative complications of STB. An epidemiological study reported that $25.7 \%$ of STB patients are complicated by pulmonary tuberculosis (18). The increased incidence of postoperative complications in STB patients with pulmonary tuberculosis may be related to the increased burden of pulmonary tuberculosis on the body. Compared with patients with STB alone, patients with multi-site tuberculosis have poorer nutritional status, weaker immune function, and less tolerance to surgery $(19,20)$, and these factors may contribute to a higher risk of overall postoperative complications.

\section{Laboratory test: preoperative serum albumin values}

This study demonstrated that preoperative serum albumin value is a risk factor for complications after spinal tuberculosis debridement, which is consistent with previous studies. Studies by Adogwa et al. and Kumar et al. found that preoperative albumin levels less than $35 \mathrm{~g} / \mathrm{L}$ is an independent risk factor for complications after elective 
Table 3 Univariate binary logistic regression analysis of risk factors between patients with and without postoperative complications

\begin{tabular}{|c|c|c|c|}
\hline Characteristics & Crude odds ratio (OR) & $95 \% \mathrm{Cl}$ & $P$ value \\
\hline Sex & 0.747 & $0.382-1.461$ & 0.394 \\
\hline BMI & 0.904 & $0.825-0.990$ & $0.030^{*}$ \\
\hline Diabetes mellitus & 1.128 & $0.017-0.963$ & $0.046^{\star}$ \\
\hline Smoking history & 1.042 & $1.005-1.081$ & $0.026^{*}$ \\
\hline Course of disease & 1.021 & $0.987-1.056$ & 0.228 \\
\hline Number of diseased vertebrae & 0.914 & $0.690-1.211$ & 0.533 \\
\hline Preoperative hemoglobin & 0.965 & $0.944-0.986$ & 0.052 \\
\hline Preoperative CRP & 1.018 & $1.002-1.034$ & $0.032^{*}$ \\
\hline Cobb angle correction & 1.056 & $1.001-1.115$ & $0.048^{*}$ \\
\hline \multicolumn{4}{|l|}{ Surgery approach } \\
\hline Anterior approach surgery & 5.786 & $2.089-16.027$ & $0.001^{*}$ \\
\hline Posterior approach surgery & 0.463 & $0.195-1.102$ & 0.082 \\
\hline Operation time & 1.018 & $1.010-1.027$ & $<0.001^{*}$ \\
\hline Operation blood loss & 1.005 & $1.002-1.007$ & $<0.001^{*}$ \\
\hline
\end{tabular}

*, $\mathrm{P}<0.05$. BMI, body mass index; CRP, C-reactive protein; OR, odds ratio.

Table 4 Multivariate binary logistic regression analysis of risk factors between patients with and without postoperative complications

\begin{tabular}{|c|c|c|c|c|}
\hline Characteristics & Adjusted odds ratio (OR) & Regression coefficients $(\beta)$ & $95 \% \mathrm{Cl}$ & $P$ value \\
\hline BMI & 0.928 & -0.079 & $0.811-1.051$ & 0.226 \\
\hline Diabetes mellitus & 1.110 & -2.211 & $0.0128-0.964$ & $0.046^{*}$ \\
\hline Pulmonary tuberculosis & 1.185 & -1.687 & $0.063-0.542$ & $0.002^{*}$ \\
\hline Preoperative serum albumin & 0.789 & -0.199 & $0.713-0.941$ & $0.005^{\star}$ \\
\hline Cobb angle correction & 0.824 & -0.006 & $0.937-1.061$ & 0.924 \\
\hline Anterior approach surgery & 5.934 & 1.781 & $1.126-26.917$ & $0.035^{\star}$ \\
\hline operation time & 1.018 & 0.018 & $1.009-1.031$ & $<0.001^{*}$ \\
\hline
\end{tabular}

*, $\mathrm{P}<0.05$. BMI, body mass index; OR, odds ratio. 

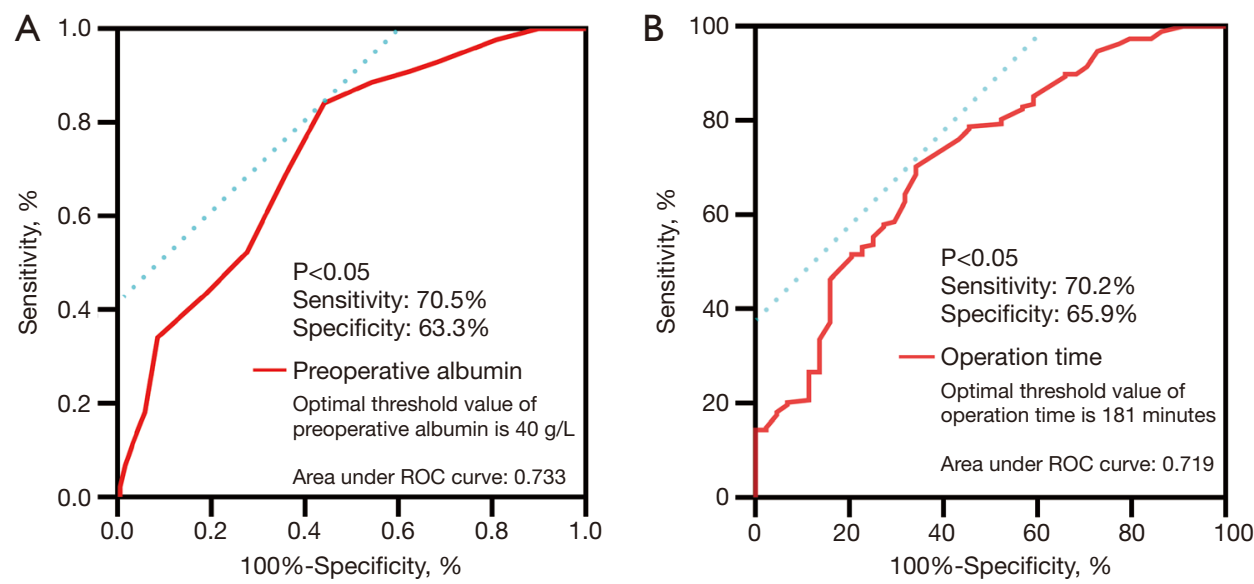

Figure 1 Threshold values for preoperative serum albumin levels (A) and operation time (B). Receiver operating characteristic (ROC) curve analysis showed the optimal threshold values of preoperative serum albumin levels and operation time were $40 \mathrm{~g} / \mathrm{L}$ and $181 \mathrm{minutes}$, respectively. ROC, receiver operating characteristic.

Table 5 Scoring system for occurrence of postoperative complications

\begin{tabular}{lc}
\hline Scoring item & Score \\
\hline Diabetes mellitus & 1 \\
Yes & 0 \\
No & \\
Pulmonary tuberculosis & 2 \\
Yes & 0 \\
No & \\
Preoperative serum albumin & 2 \\
$\leq 40$ & 0 \\
$>40$ & \\
Anterior approach surgery & 1 \\
Yes & 0 \\
No & \\
Operation time & \\
181 & 0 \\
\hline 181 & 3 \\
\hline
\end{tabular}

degenerative and malformed spinal fusion $(21,22)$. In contrast, this current study determined that preoperative albumin values less than $40 \mathrm{~g} / \mathrm{L}$ is an independent risk factor for overall postoperative complications. Two reasons may explain the higher preoperative serum albumin requirements in patients with STB. First, patients

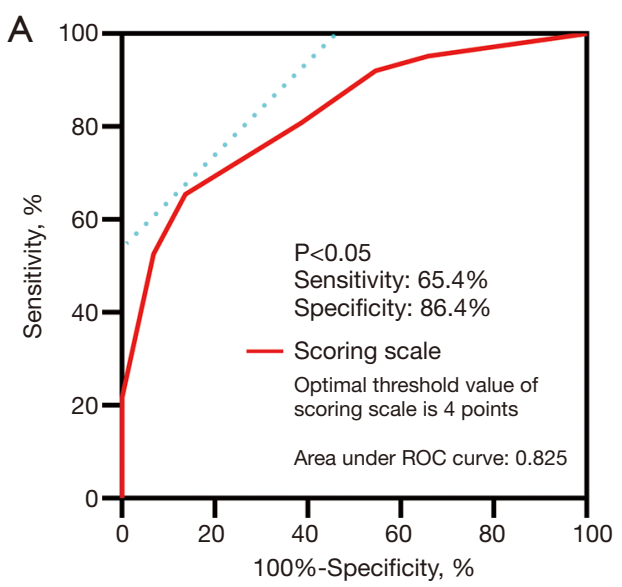

Figure 2 Receiver operating characteristic (ROC) curve analysis of the scoring scale. The optimal cut-off point based on ROC curve analysis was 4 points. ROC, receiver operating characteristic.

with STB are prone to be malnourished, and disease and surgery can increase the likelihood of postoperative complications (18). Second, STB debridement causes more blood loss and requires longer operation times than spinal degenerative disease surgery, and thus patients are more likely to experience complications such as hypoalbuminemia after surgery with a greater decrease in postoperative albumin (22). Although preoperative albumin is associated with an increased incidence of postoperative complications, the precise complications and the mechanisms involved remain unclear and warrant further research. 
Table 6 Comparison of performance of the scoring scale on derivation set and validation set

\begin{tabular}{|c|c|c|c|c|c|c|}
\hline Groups & \multicolumn{3}{|c|}{ Derivation set } & \multicolumn{3}{|c|}{ Validation set } \\
\hline \multicolumn{7}{|c|}{ Clinical diagnosis } \\
\hline With POC & 123 & 64 & 187 & 33 & 17 & 50 \\
\hline Without POC & 6 & 39 & 45 & 1 & 11 & 12 \\
\hline Sensitivity (\%) & & 65.4 & & & 60.8 & \\
\hline Specificity (\%) & & 86.4 & & & 81.8 & \\
\hline
\end{tabular}

POC, postoperative complication.

Table 7 Incidence of POC's occurrence with different scores

\begin{tabular}{lccc}
\hline Score & Patients with POC & Total patients & Incidence of POC (\%) \\
\hline 0 & 9 & 24 & 37.5 \\
1 & 6 & 11 & 54.5 \\
2 & 19 & 28 & 67.9 \\
3 & 30 & 40 & 75.0 \\
4 & 25 & 27 & 92.6 \\
5 & 58 & 61 & 95.1 \\
6 & 9 & 9 & 100 \\
7 & 27 & 27 & 100 \\
8 & 4 & 4 & 100 \\
9 & 1 & 1 & 100 \\
\hline
\end{tabular}

POC, postoperative complication.

\section{Operation-related findings: operation time and surgical approach}

Studies have shown that operation time is related to the rate of postoperative incision infections after spinal surgery $(23,24)$. Furthermore, intraoperative blood loss, surgical trauma, and duration of anesthesia are risk factors of postoperative complications (24-26). In this current study, operation time, which can be used to reflect intraoperative blood loss, surgical trauma, and duration of anesthesia, was shown to be an independent risk factor of overall postoperative complications. The average operation time of patients who presented with postoperative complications was $212.21 \pm 56.11$ minutes compared to $169.93 \pm 46.26$ minutes in patients who did not experience postoperative complications $(\mathrm{P}<0.001)$. Analysis using the ROC curve determined that operation time of 181 minutes was the diagnostic threshold for the occurrence of postoperative complications. In a study addressing the risk of spinal infections, Beiner et al. suggested that an operation time longer than 180 minutes predisposes the patients to postoperative infections, and antibiotics should be administered when surgery lasts longer than 180 minutes (27). The results of the latter study concur with our data and, taken together, suggest that controlled operation time within 180 minutes can reduce the risk of postoperative incision infections and the risk of overall postoperative complications. Therefore, optimizing operation time in STB patients to 180 minutes or less may be a crucial step in minimizing postoperative complications.

The anterior and posterior surgical approaches are commonly used in STB focus debridement. Our study indicated that using the anterior approach is an independent 


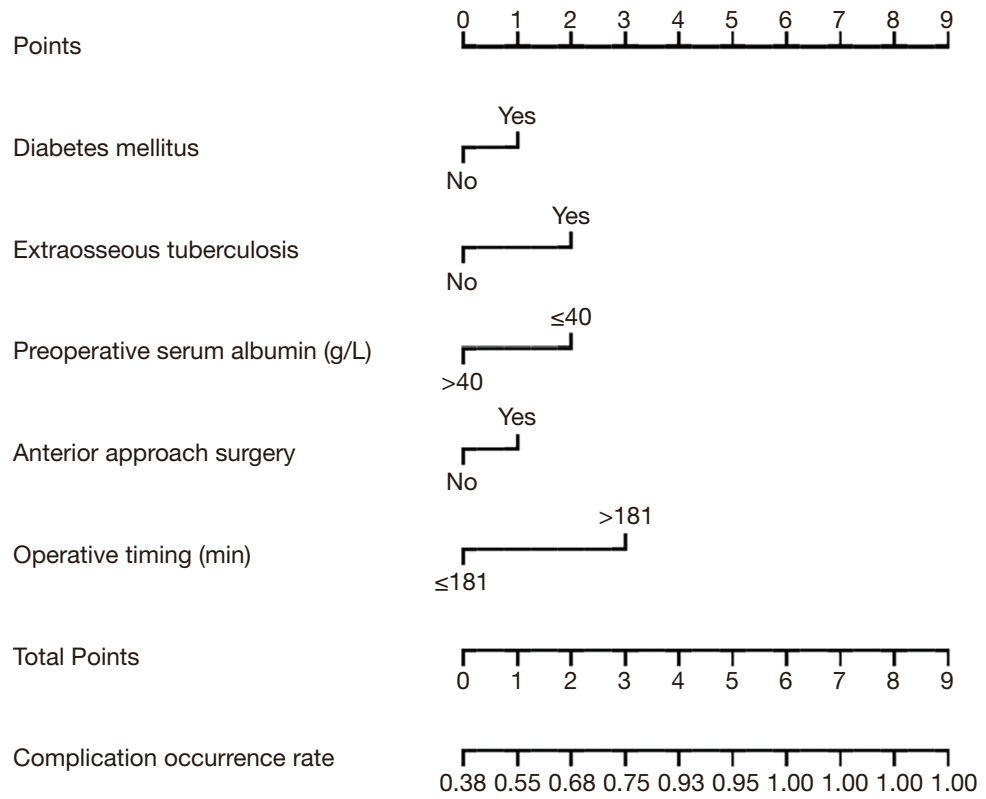

Figure 3 Nomogram for predicting postoperative complications (C-index: 0.825) after STB debridement. The rate of complications was estimated by adding the scores of diabetes mellitus, pulmonary tuberculosis, preoperative serum albumin, anterior approach surgery, and operation time. STB, spinal tuberculosis.

risk factor of postoperative complications, while the posterior approach is not a risk factor. Interestingly, the anterior approach is superior to the posterior approach regarding operative time, intraoperative bleeding, and correction of the Cobb angle $(28,29)$. Furthermore, the anterior approach can achieve adequate debridement, better decompression, less muscle injury, and better interbody fusion cage placement. However, it cannot provide a satisfactory orthopedic result, causes more serious postoperative pain, and results in more complications related to the chest and abdomen due to the surgical channel (30). The risk of postoperative intestinal obstruction is $7.0 \%$ in anterior lumbar spinal surgeries (29).

On the contrary, the posterior surgical approach has the advantages of better implant fixation and better kyphosis correction. However, it is associated with insufficient nerve decompression and lesion removal, increased intraoperative bleed, and longer operative times (29). In summary, the anterior approach has better decompression effects but increased risk of postoperative complications, while the posterior approach has better orthopedic effects and fewer postoperative complications.

\section{Scoring scale}

STB patients have poor immunity, severe surgical trauma, and a high risk of postoperative complications $(6,25,31)$. The development of a scoring scale to predict postoperative complications after STB debridement surgery is vital for the perioperative management of patients and can effectively help physicians decide on postoperative treatment strategies. This study is the first to quantify the risk of postoperative complications in spinal tuberculosis to the best of our knowledge. However, there were some limitations to the development of the scoring scale. Due to the small sample size of the validation set, the diagnostic accuracy of the scale cannot be fully validated. As the scale's sensitivity was not high, there may be a significant rate of missed diagnosis. In addition, there may have been other independent clinical risk factors that were not included in the present study. Further studies are warranted to confirm the validity and improve upon the scoring scale for risk assessment of postoperative complications in patients with STB.

\section{Conclusions}

This investigation identified five independent risk factors for postoperative complications in patients with STB, which were subsequently used to develop a scoring scale. A score of more than 4 points is considered to be associated with postoperative complications with high sensitivity and 
specificity. This scale is mainly dependent on clinical data, which is suitable for doctors to predict the changes in a patient's postoperative condition.

\section{Study limitations}

There were some limitations in this investigation. First, there may be some risk factors we didn't take into inclusion. Second, the risk factors for different levels of postoperative complications were not investigated (32). Third, the sample size of the validation set was small. Future studies addressing these issues will be required to confirm these results.

\section{Acknowledgments}

Funding: This study was supported by the Natural Science Foundation of Chongqing (Grant Number: cstc2019jcyjmsxmX0358).

\section{Footnote}

Reporting Checklist: The authors have completed the TRIPOD reporting checklist. Available at https://dx.doi. org/10.21037/apm-21-851

Data Sharing Statement: Available at https://dx.doi. org/10.21037/apm-21-851

Conflicts of Interest: All authors have completed the ICMJE uniform disclosure form (available at https://dx.doi. org/10.21037/apm-21-851). The authors have no conflicts of interest to declare.

Ethical Statement: The authors are accountable for all aspects of the work in ensuring that questions related to the accuracy or integrity of any part of the work are appropriately investigated and resolved. This study was conducted in accordance with the Declaration of Helsinki (as revised in 2013) and was approved by the Institutional Ethics Board of The First Affiliated Hospital of Chongqing Medical University (No. ChiCTR1800019109). All participants provided written informed consent to participate in this study.

Open Access Statement: This is an Open Access article distributed in accordance with the Creative Commons Attribution-NonCommercial-NoDerivs 4.0 International
License (CC BY-NC-ND 4.0), which permits the noncommercial replication and distribution of the article with the strict proviso that no changes or edits are made and the original work is properly cited (including links to both the formal publication through the relevant DOI and the license). See: https://creativecommons.org/licenses/by-nc-nd/4.0/.

\section{References}

1. Dunn RN, Ben Husien M. Spinal tuberculosis: review of current management. Bone Joint J 2018;100-B:425-31.

2. Wang YX, Zhang HQ, Li M, et al. Debridement, interbody graft using titanium mesh cages, posterior instrumentation and fusion in the surgical treatment of multilevel noncontiguous spinal tuberculosis in elderly patients via a posterior-only. Injury 2017;48:378-83.

3. Boachie-Adjei O, Papadopoulos EC, Pellisé F, et al. Late treatment of tuberculosis-associated kyphosis: literature review and experience from a SRS-GOP site. Eur Spine J 2013;22 Suppl 4:641-6.

4. Rajasekaran S, Soundarapandian S. Progression of kyphosis in tuberculosis of the spine treated by anterior arthrodesis. J Bone Joint Surg Am 1989;71:1314-23.

5. Swann MC, Hoes KS, Aoun SG, et al. Postoperative complications of spine surgery. Best Pract Res Clin Anaesthesiol 2016;30:103-20.

6. Rihn JA, Patel R, Makda J, et al. Complications associated with single-level transforaminal lumbar interbody fusion. Spine J 2009;9:623-9.

7. Zhang $\mathrm{H}$, Yin $\mathrm{X}, \mathrm{Li} \mathrm{F}$, et al. Investigation of the complications associated with surgery for treating spinal tuberculosis. Orthopedic Journal of China 2014;22:20-7.

8. Wang X, Zhou B, Li W, et al. Cause analysis and prevention of the complications after surgical treatment for spinal tuberculosis. Chinese Journal of Spine and Spinal Cord 2010;20:993-7.

9. Wong AP, Smith ZA, Nixon AT, et al. Intraoperative and perioperative complications in minimally invasive transforaminal lumbar interbody fusion: a review of 513 patients. J Neurosurg Spine 2015;22:487-95.

10. Wang H, Yang X, Shi Y, et al. Early predictive factors for lower-extremity motor or sensory deficits and surgical results of patients with spinal tuberculosis: A retrospective study of 329 patients. Medicine (Baltimore) 2016;95:e4523.

11. Zeng ZY, Xu ZW, He DW, et al. Complications and Prevention Strategies of Oblique Lateral Interbody Fusion Technique. Orthop Surg 2018;10:98-106. 
12. Bao YC, Yu M, Tang L, et al. Changes in Serum Prealbumin and Incision Complications Following Spinal Tuberculosis Surgery: A Preliminary Study. Orthop Surg 2021;13:501-5.

13. Kharbanda AB, Taylor GA, Fishman SJ, et al. A clinical decision rule to identify children at low risk for appendicitis. Pediatrics 2005;116:709-16.

14. Zhou X, Qiao Q, Ji L, et al. Nonlaboratory-based risk assessment algorithm for undiagnosed type 2 diabetes developed on a nation-wide diabetes survey. Diabetes Care 2013;36:3944-52.

15. Browne JA, Cook C, Pietrobon R, et al. Diabetes and early postoperative outcomes following lumbar fusion. Spine (Phila Pa 1976) 2007;32:2214-9.

16. Sharma A, Muir R, Johnston R, et al. Diabetes is predictive of longer hospital stay and increased rate of complications in spinal surgery in the UK. Ann R Coll Surg Engl 2013;95:275-9.

17. Gautam MP, Karki P, Rijal S, et al. Pott's spine and paraplegia. JNMA J Nepal Med Assoc 2005;44:106-15.

18. Wang H, Li C, Wang J, et al. Characteristics of patients with spinal tuberculosis: seven-year experience of a teaching hospital in Southwest China. Int Orthop 2012;36:1429-34.

19. Gupta KB, Gupta R, Atreja A, et al. Tuberculosis and nutrition. Lung India 2009;26:9-16.

20. Karyadi E, Schultink W, Nelwan RH, et al. Poor micronutrient status of active pulmonary tuberculosis patients in Indonesia. J Nutr 2000;130:2953-8.

21. Adogwa O, Martin JR, Huang K, et al. Preoperative serum albumin level as a predictor of postoperative complication after spine fusion. Spine (Phila Pa 1976) 2014;39:1513-9.

22. Kumar S, van Popta D, Rodrigues-Pinto R, et al. Risk factors for wound infection in surgery for spinal metastasis. Eur Spine J 2015;24:528-32.

23. Kudo D, Miyakoshi N, Hongo M, et al. Relationship

Cite this article as: Jiang G, Ou Y, Zhu Y, Luo W, Du X, Zhang W, Qin W. Development of a scoring scale for predicting the risk of postoperative complications after spinal tuberculosis debridement: a retrospective cohort study of 233 patients. Ann Palliat Med 2021;10(9):9372-9382. doi: 10.21037/ apm-21-851 between preoperative serum rapid turnover proteins and early-stage surgical wound infection after spine surgery. Eur Spine J 2017;26:3156-61.

24. Ialenti MN, Lonner BS, Verma K, et al. Predicting operative blood loss during spinal fusion for adolescent idiopathic scoliosis. J Pediatr Orthop 2013;33:372-6.

25. Zhou B, Li W, Sun C, et al. Risk factors for multiple debridements of the patients with deep incisional surgical site infection after spinal surgery.Journal of Peking University (Health Sciences) 2021:1-15.

26. Fedok FG, Ferraro RE, Kingsley CP, et al. Operative times, postanesthesia recovery times, and complications during sinonasal surgery using general anesthesia and local anesthesia with sedation. Otolaryngol Head Neck Surg 2000;122:560-6.

27. Beiner JM, Grauer J, Kwon BK, et al. Postoperative wound infections of the spine. Neurosurg Focus 2003;15:E14.

28. Zheng B, Hao D, Guo H, et al. Anterior versus posterior surgical approach for lumbosacral tuberculosis. J Int Med Res 2018;46:2569-77.

29. Hassan K, Elmorshidy E. Anterior versus posterior approach in surgical treatment of tuberculous spondylodiscitis of thoracic and lumbar spine. Eur Spine J 2016;25:1056-63.

30. Al Maaieh MA, Du JY, Aichmair A, et al. Multivariate analysis on risk factors for postoperative ileus after lateral lumbar interbody fusion. Spine (Phila Pa 1976) 2014;39:688-94.

31. Danaviah S, Sacks JA, Kumar KP, et al. Immunohistological characterization of spinal TB granulomas from HIVnegative and -positive patients. Tuberculosis (Edinb) 2013;93:432-41.

32. Dindo D, Demartines N, Clavien PA. Classification of surgical complications: a new proposal with evaluation in a cohort of 6336 patients and results of a survey. Ann Surg 2004;240:205-13. 